\title{
COMPONENTS IN VECTOR LATTICES AND EXTREME EXTENSIONS OF QUASI-MEASURES AND MEASURES
}

\author{
by Z. LIPECKI
}

(Received 25 October, 1991)

1. Introduction. We develop some ideas contained in the author's paper [8] which was, in turn, inspired by Bierlein and Stich [5]. The main body of the present paper $\dagger$ is divided into three sections. Section 2 is concerned with some vector-lattice-theoretical results. They are then applied to extensions of quasi-measures and measures in Sections 3 and 4 , respectively.

Let $X$ be a vector lattice, let $x \in X_{+}$and let $S$ be a non-empty set. Theorems 1 and 2 describe some properties of the convex set

$$
\mathscr{D}_{x, s}=\left\{\left(\xi^{s}\right) \in X^{s}: \xi^{s} \geqslant 0 \text { for all } s \in S \text { and } \sum_{s \in S} \xi^{s}=x\right\}
$$

(see Section 2 for the definition of the sum above). The extreme points of $\mathscr{D}_{x, s}$ are characterized in terms of the components of $x$. It is also shown that if $X$ has the principal projection property and $S$ is countable, then extr $\mathscr{D}_{x, s}$ is, in some sense, large in $\mathscr{D}_{x, s}$. Furthermore, for finite $S$, each point in $\mathscr{D}_{x, S}$ is then a $\sigma$-convex combination of extreme ones.

Let $\mathfrak{M}$ and $\mathfrak{R}$ be algebras of subsets of a set $\Omega$ with $\mathfrak{M} \subset \mathfrak{R}$. We denote by ba( $\mathfrak{M})$ the vector lattice of all real-valued bounded additive functions on $\mathfrak{M}$. For a quasi-measure $\mu$ on $\mathfrak{M}$, i.e. an element of $b a_{+}(\mathfrak{M})$, we define

$$
E(\mu)=\left\{\rho \in \mathrm{ba}_{+}(\mathfrak{R}): \rho \mid \mathfrak{M}=\mu\right\} .
$$

We give a description of $E(\mu)$ in the case where $\mathfrak{R}$ is generated by $\mathfrak{M}$ and an $n$-element partition of $\Omega$. This description allows us to identify $E(\mu)$ with an extreme subset (= face) of $\mathscr{D}_{\mu, S}$ for $S$ of cardinality $n$ and, consequently, to apply the material of Section 2 (see Theorem 3).

Let now $\mathfrak{M}$ and $\mathfrak{R}$ be $\sigma$-algebras of subsets of $\Omega$ with $\mathfrak{M} \subset \mathfrak{R}$. We denote by ca(M) the vector lattice of all real-valued $\sigma$-additive functions on $\mathfrak{M}$. For a measure $\mu$ on $\mathfrak{M}$, i.e. an element of $\mathrm{ca}_{+}(\mathfrak{M})$, we define

$$
E_{\sigma}(\mu)=E(\mu) \cap \mathrm{ca}(\Re) .
$$

We give a description of $E_{\sigma}(\mu)$ in the case where $\mathfrak{R}$ is generated by $\mathfrak{M}$ and a countable partition of $\Omega$. This description allow us to identify $E_{\sigma}(\mu)$ with an extreme subset of $\mathscr{D}_{\mu, \mathbb{N}}$ and, consequently, to apply the material of Section 2 (see Theorem 6 and the proof of Theorem 7). The results obtained are closely related to some theorems in Bierlein [4] and Bierlein and Stich [5].

In Sections 3 and 4 we also examine the problem whether each element of $E(\mu)$ or $E_{\sigma}(\mu)$ dominates, in the sense of absolute continuity, an extreme one (see the affirmative Theorems 5 and 7 and the negative Examples 2 and 3). The problem seems to have been mentioned for the first time in [8].

$\dagger$ Some results of the paper were presented at the conference "Topology and Measure VI", Warnemünde (Germany), August 1991.

Glasgow Math. J. 35 (1993) 153-162. 
Throughout the paper we dispense with uncountable forms of the axiom of choice. The only exception is the proof of Theorem 5, where the Stone representation theorem and the non-effective part of Plachky's criterion [12] are applied.

The vector-lattice-theoretical notation and definitions we use are gathered at the beginning of Section 2. Sections 3 and 4 start with the explanation of our notation concerning quasi-measures and measures, respectively.

2. Components and extreme points in vector lattices. Throughout this section $X$ denotes a vector lattice (or a Riesz space in the terminology of [1] and [9]) over the reals $\mathbb{R}$ and $X_{+}$stands for its positive cone. Given $x \in X_{+}$, we put

$$
\mathscr{C}_{x}=\{y \in X: y \wedge(x-y)=0\} .
$$

The elements of $\mathscr{C}_{x}$ are called components of $x$ ([1], p. 36, or [9], Definition 38.1). The set $\mathscr{C}_{x}$ is a sublattice of $X$ with smallest element 0 and greatest element $x$. For $y \in \mathscr{C}_{x}$ we have $y \vee(x-y)=x$, which shows that $x-y$ is a complement of $y$ in $\mathscr{C}_{x}$. Thus, $\mathscr{C}_{x}$ is a Boolean algebra (see [1], Theorem 3.15, for details). The difference operation in $\mathscr{C}_{x}$ is denoted by $\backslash$, that is

$$
y_{1} \backslash y_{2}=y_{1} \wedge\left(x-y_{2}\right) \text { for } y_{1}, y_{2} \in \mathscr{C}_{x} .
$$

Following [1], pp. 30-31, we denote by $B_{x}$ the band generated by $x$ in $X$. If $B_{x}$ is a projection band ([1], p. 32), $P_{x}$ stands for the (band) projection of $X$ onto $B_{x}$. Clearly, we then have $P_{x}(y) \in \mathscr{C}_{y}$ for every $y \in X_{+}$. We say that $X$ has the principal projection property, PPP for short, provided that $B_{x}$ is a projection band for every $x \in X([1], p .35$, or [9], Definition 24.8). Every Dedekind complete vector lattice has PPP (see [1], Theorem 3.7, or [9], Theorem 25.1).

In the sequel $S$ stands for an arbitrary set consisting of at least two elements. We consider $X^{S}$ as a vector lattice with the usual product ordering ([1], p. 18). The elements of $X^{S}$ are denoted by boldface small Greek letters $\xi, \eta, \zeta$. Their coordinates are denoted by ordinary small Greek letters equipped with superscripts, e.g. $\xi=\left(\xi^{s}\right)$. For $\xi \in\left(X^{S}\right)_{+}$ we define

$$
\sum_{s \in S} \xi^{s}=\sup \left\{\sum_{s \in S_{0}} \xi^{s}: S_{0} \text { is a finite subset of } S\right\}
$$

provided the supremum exists. We say that a subset $W$ of $X_{+}$is $\sigma$-convex if it is closed under taking $\sigma$-convex combinations of its elements, i.e. for all $t_{j}>0$ with $\sum_{j=1}^{\infty} t_{j}=1$ and all $\xi \in W^{\mathbb{N}}$ we have $\sum_{j=1}^{\infty} t_{j} \xi^{j} \in W$ provided the sum exists.

Given $x \in X_{+}$, we set

$$
\begin{gathered}
\mathscr{D}_{x, S}=\left\{\xi \in\left(X^{s}\right)_{+}: \sum_{s \in S} \xi^{s}=x\right\}, \\
\mathscr{C}_{x, S}=\left\{\xi \in \mathscr{D}_{x, S}: \xi^{s} \wedge \xi^{s^{\prime}}=0 \text { whenever } s, s^{\prime} \in S \text { and } s \neq s^{\prime}\right\} .
\end{gathered}
$$

In the case where $S=\{1, \ldots, n\}$ we write $X^{n}, \mathscr{D}_{x, n}$ and $\mathscr{C}_{x, n}$ for short.

THEOREM 1. (a) $\mathscr{C}_{x, S} \subset \mathscr{D}_{x, S}$ and $\mathscr{D}_{x, S}$ is a $\sigma$-convex subset of $X^{S}$.

(b) extr $\mathscr{D}_{x, S}=\mathscr{C}_{x, S}$. 
Part (b) has been previously known in several special cases. In fact, the case where $S$ has exactly two elements is equivalent to the following assertion contained in [1], Theorem 3.15: $\operatorname{extr}[0, x]=\mathscr{C}_{x}$. (The inclusion " $\supset$ " of the latter assertion is used in the proof of (b) below.) The case where $X=L^{\infty}(\mu)$ with a probability measure $\mu, x$ is the constant 1 function, and $S$ is finite or countable can be found in [6], Corollary 2.3 and Theorem 5.2 (cf. also [7], proof of Theorem 5). Finally, for $X=\mathbb{R}^{m}, x=(1, \ldots, 1)$ and $S=\{1, \ldots, n\}$ the set $\mathscr{D}_{x, S}$ can be interpreted as the set of all stochastic matrices with $m$ rows and $n$ columns. According to (b), a stochastic matrix is extreme if and only if its entries are all equal to 0 or 1 (cf. [1], Section 7, Exercise 7).

Proof of Theorem 1. Only part (b) needs a proof. For $\xi \in \mathscr{C}_{x, S}$ we have

$$
\xi^{s} \wedge \sum_{s^{\prime} \in S \backslash\{s\}} \xi^{s^{\prime}}=0 \text { and } \xi^{s}+\sum_{s^{\prime} \in S \backslash\{s\}} \xi^{s^{\prime}}=x
$$

whence $\xi^{s} \in \mathscr{C}_{x}$ for all $s \in S$. Suppose $\xi=t \boldsymbol{\eta}_{1}+(1-t) \boldsymbol{\eta}_{2}$, where $\boldsymbol{\eta}_{1}, \boldsymbol{\eta}_{2} \in \mathscr{D}_{x, s}$ and $0<t<1$. Then $\xi^{s}=t \eta_{1}^{s}+(1-t) \eta_{2}^{s}$, whence $\xi^{s}=\eta_{1}^{s}=\eta_{2}^{s}$, by the inclusion $\mathscr{C}_{x} \subset \operatorname{extr}[0, x]$ mentioned above.

Now, let $\xi \in \mathscr{D}_{x, s}$ and $\xi^{s^{\prime}} \wedge \xi^{s^{\prime \prime}} \neq 0$ for some distinct $s^{\prime}, s^{\prime \prime} \in S$. Set $y=\xi^{s^{\prime}} \wedge \xi^{s^{\prime \prime}}$, and define $\boldsymbol{\eta} \in \dot{X}^{S}$ by

$$
\eta^{s}=\left\{\begin{array}{rll}
0 & \text { if } & s \neq s^{\prime}, s^{\prime \prime} \\
y & \text { if } & s=s^{\prime} \\
-y & \text { if } & s=s^{\prime \prime}
\end{array}\right.
$$

Then $\boldsymbol{\xi} \pm \boldsymbol{\eta} \in \mathscr{D}_{x, s}$, whence $\xi \notin$ extr $\mathscr{D}_{x, s}$

We define

$$
t_{n j}=\frac{1}{n}\left(1-\frac{1}{n}\right)^{j-1}, \quad n=2,3, \ldots, j=1,2, \ldots
$$

We have $t_{n j}>0$ and $\sum_{j=1}^{\infty} t_{n j}=1$. The coefficients $t_{n j}$ appear in Theorems 2(b) and 3(d) below. They have already been used in [5], proof of Theorem 3, in a similar context.

Theorem 1(b) shows that $\mathscr{D}_{x, s}$ has always extreme points. This is seen by considering the points $\xi \in \mathscr{D}_{x, S}$ such that $\xi^{s}=x$ for some $s \in S$ (and $\xi^{s}=0$ for other $s \in S$ ). The next result shows that, under some additional assumptions on $X$ and $S$, the set $\mathscr{D}_{x, S}$ has sufficiently many extreme points for our purposes. In connection with part (a) note that $B_{\xi}$ stands for the band generated by $\xi$ in $X^{S}$. Part (b) originates with a theorem due to R. Bierlein ([5], Theorem 3). For $n=2$ this part reduces to [8], Theorem 4; see also [11] for a related result.

Theorem 2. Suppose $X$ has $P P P$ and $x \in X_{+}$. Then

(a) For every $\xi \in \mathscr{D}_{x, \mathbb{N}}$ there exists $\boldsymbol{\eta} \in \mathscr{C}_{X, \mathbb{N}}$ with $\boldsymbol{\eta} \in B_{\xi}$;

(b) For every $\xi \in \mathscr{D}_{x, n}$ there exist $\boldsymbol{\eta}_{j} \in \mathfrak{C}_{x, n}$ with $\xi=\sum_{j=1}^{\infty} t_{n j} \boldsymbol{\eta}_{j}$;

(c) For every $\xi \in\left(X^{2}\right)_{+}$with $x \leqslant \xi^{1}+\xi^{2}$ there exists $\eta \in \mathscr{C}_{x .2}$ with $\eta^{\prime} \in B_{\xi^{\prime}}$ and $\eta^{2} \leqslant \xi^{2}$ 
Proof. (a) Put $\zeta^{i}=P_{\xi i}(x)$. Then $\bigvee_{i=1}^{\infty} \zeta^{i}=x$. Indeed, denoting $y_{k}=\bigvee_{i=1}^{k} \xi^{i}$ and $z_{k}=$ $\sum_{i=1}^{k} \xi^{i}$, we have (see [9], p. 181)

$$
x \geqslant \bigvee_{i=1}^{k} \zeta^{i}=P_{y_{k}}(x)=P_{z_{k}}(x) \geqslant \sum_{i=1}^{k} \xi^{i}
$$

We have $\zeta^{i} \in \mathscr{C}_{x}$, and so, taking into account that $\mathscr{C}_{x}$ is a Boolean algebra, we can define

$$
\eta^{1}=\zeta^{1}, \eta^{i+1}=\zeta^{i+1} \backslash \bigvee_{j=1}^{i} \zeta^{i}
$$

Then $\eta^{i} \in \mathscr{C}_{x}$ and $\eta^{i} \wedge \eta^{i^{\prime}}=0$ whenever $i, i^{\prime} \in \mathbb{N}$ and $i \neq i^{\prime}$. Moreover, $\eta^{i} \in B_{\xi^{i}}$, since $0 \leqslant \eta^{i} \leqslant \zeta^{i}$. Finally,

$$
x \geqslant \sum_{i=1}^{k} \eta^{i}=\bigvee_{i=1}^{k} \eta^{i}=\bigvee_{i=1}^{k} \zeta^{i}
$$

and so $\sum_{i=1}^{\infty} \eta^{i}=x$. Therefore $\boldsymbol{\eta} \in \mathscr{C}_{x, \mathbb{N}}$.

(b) We first observe that

$$
\sum_{i=1}^{n}\left(\xi^{i}-\sum_{j=1}^{k} t_{n j} \eta_{j}^{i}\right)=n t_{n, k+1} x, \quad k=1,2, \ldots
$$

whenever $\boldsymbol{\eta}_{1}, \ldots, \boldsymbol{\eta}_{k} \in \mathscr{D}_{x, n}$. Indeed, we have

$$
1-\sum_{j=1}^{k} t_{n j}=\left(1-\frac{1}{n}\right)^{k}=n t_{n, k+1}
$$

We shall define, by induction, $\boldsymbol{\eta}_{j} \in \mathscr{C}_{x, n}$ such that

$$
\xi \geqslant \sum_{j=1}^{k} t_{n j} \boldsymbol{\eta}_{j}, \quad k=1,2, \ldots
$$

Then the $\eta_{j}$ 's are as desired. This is seen, since (1) and (2) imply

$$
0 \leqslant \xi^{i}-\sum_{j=1}^{k} t_{n j} \eta_{j}^{i} \leqslant n t_{n, k+1} x
$$

and $X$ is Archimedean ([9], Theorem 25.1).

In order to define $\boldsymbol{\eta}_{1}$, it is enough to find $\boldsymbol{\eta} \in\left(\mathscr{C}_{x}\right)^{n}$ with $\bigvee_{i=1}^{n} \eta^{i}=x$ and $\xi \geqslant$ $\frac{1}{n} \eta$, and then use the standard disjointization procedure (see the proof of (a) above). Let $P_{i}$ stand for the projection on the band generated by $\left(x-n \xi^{i}\right)_{+}$in $X$. Define

$$
\eta^{i}=x-P_{i}(x), \quad i=1, \ldots, n \text {. }
$$

Since $\xi \in \mathscr{D}_{x, n}$, we have

$$
\bigwedge_{i=1}^{n}\left(x-n \xi^{i}\right)_{+}=0, \text { whence } \bigvee_{i=1}^{n} \eta^{i}=x
$$

On the other hand, $x \geqslant\left(x-n \xi^{i}\right)_{+}$implies $P_{i}(x) \geqslant x-n \xi^{i}$, i.e. $n \xi^{i} \geqslant \eta^{i}$. 
Suppose now (2) holds for some $k$, and define

$$
\zeta=\frac{1}{n}\left(t_{n, k+1}\right)^{-1}\left(\xi-\sum_{j=1}^{k} t_{n j} \boldsymbol{\eta}_{j}\right) .
$$

Then, in view of (1), we get $\zeta \in \mathscr{D}_{x, n}$. Applying (2) for $k=1$, we get $\boldsymbol{\eta}_{k+1} \in \mathscr{C}_{x, n}$ with

$$
\zeta-\frac{1}{n} \boldsymbol{\eta}_{k+1} \geqslant 0
$$

and the induction step is accomplished.

(c) Define $\eta^{1}=P_{\xi^{\prime}}(x)$ and $\eta^{2}=x-\eta^{1}$. Since $\eta^{2} \wedge \xi^{1}=0$ and $\eta^{2} \leqslant \xi^{1}+\xi^{2}$, we have $\eta^{2} \leqslant \xi^{2}$.

Of course, (c) cannot be strengthened to the effect that $\eta \in \mathscr{C}_{x, 2}$ and $\eta \leqslant \xi$. (Take $X=\mathbb{R}, x=1$ and $\xi=\left(\frac{1}{2}, \frac{1}{2}\right)$.) Modifying an example due to $\mathrm{R}$. Bierlein ([5], pp. 95-96), we shall show that for $\xi \in \mathscr{D}_{x, \mathbb{N}}$ there may be no $\boldsymbol{\eta} \in \mathscr{C}_{x, \mathbb{N}}$ and $t>0$ with $t \boldsymbol{\eta} \leqslant \xi$. Therefore (a) and (b) are, in some sense, best possible.

Example 1. Take $X=\mathbb{R}^{\mathbb{N}}$ and $x=1_{\mathbb{N}}$. Then $\mathscr{C}_{x}=\{0,1\}^{\mathbb{N}}$. Define

$$
\xi^{i}=\frac{1}{k} 1_{\{k-1\}} \quad \text { if } \quad \frac{(k-1) k}{2} \leqslant i<\frac{k(k+1)}{2}, \quad k=2,3, \ldots
$$

Clearly, $\xi \in \mathscr{D}_{x, \mathbb{N}}$. Moreover, $\boldsymbol{\eta} \in \mathscr{C}_{x, \mathbb{N}}$ if and only if there exists a partition $\left\{E_{1}, E_{2}, \ldots\right\}$ of $\mathbb{N}$ with $\eta^{i}=1_{E_{i}}$ for all $i$. Therefore $t \boldsymbol{\eta} \leqslant \xi$ implies $t \leqslant 0$.

3. Extensions of quasi-measures. Throughout this section $\mathfrak{M}$ and $\mathfrak{R}$ stand for algebras of subsets of a set $\Omega$ with $\mathfrak{M} \subset \mathfrak{R}$. Given $\mathfrak{U}_{\subset} \subset 2^{\Omega}$, we denote by $\mathfrak{U}_{b}\left[\mathfrak{U}_{\beta}\right]$ the algebra [ $\sigma$-algebra] of subsets of $\Omega$ generated by $\mathbb{U}$. The Dedekind complete vector lattice of all real-valued bounded additive functions on $\mathfrak{M}$ is denoted by ba( $(\mathfrak{R})$ (see [3], Section 2.2). The elements of $\mathrm{ba}_{+}(\mathfrak{M})$ are called quasi-measures. For $\mu, v \in \mathrm{ba}_{+}(\mathfrak{M})$ we write $\mu \ll v$ if $\mu$ is $(\varepsilon-\delta)$ absolutely continuous with respect to $v$ (see [3], Definition 6.1.1).

Let $\mu \in \mathrm{ba}_{+}(\mathfrak{M})$. The ideal of $\mu$-null sets in $\mathfrak{M}$ is denoted by $\mathcal{N}(\mu)$. Moreover, we denote by $E(\mu, \mathfrak{R})$ or $E(\mu)$ the $\sigma$-convex set

$$
\left\{\rho \in \mathrm{ba}_{+}(\Re): \rho \mid \mathfrak{M}=\mu\right\} .
$$

It is well known that $E(\mu)$ is always non-empty (see [3], Theorem 3.4.4).

Generalizing a part of [8], Theorem 1, we now give a description of $E(\mu)$ in a special case. This description allows us to apply Theorems 1 and 2 above. As a result, we obtain a generalization of the remaining part of [8], Theorem 1, and an improvement of [8], Theorem 3. The advantage of the present method over the one used in [8] is that no appeal is made to uncountable forms of the axiom of choice.

Theorem 3. Let $\mu \in \mathrm{ba}_{+}(\mathfrak{M})$ and $\mathfrak{M}=\left(\mathfrak{M} \cup\left\{E_{1}, \ldots, E_{n}\right\}\right)_{b}$, where $\left\{E_{1}, \ldots, E_{n}\right\}$ is $a$ partition of $\Omega$. Define $\mathscr{E}_{\mu, n}$ as $\left\{\boldsymbol{\rho} \in \mathscr{D}_{\mu, n}: M \cap E_{i}=\varnothing\right.$ implies $\rho^{i}(M)=0$ whenever $M \in \mathfrak{M}$, $i=1, \ldots, n\}$. Then

(a) $\mathscr{C}_{\mu, n}$ is an extreme subset of $\mathscr{D}_{\mu, n}$.

(b) The mapping $\rho \rightarrow \rho$ of ba $_{+}(\mathfrak{R})$ into $\left(\mathrm{ba}_{+}(\mathfrak{M})\right)^{n}$, where $\rho^{i}(M)=\rho\left(M \cap E_{i}\right)$ for all $M \in \mathfrak{M}$ and $i=1, \ldots, n$, is injective, preserves $\sigma$-convex combinations and takes $E(\mu)$ onto $\mathscr{E}_{\mu, n}$. Moreover, $\rho_{1} \ll \rho_{2}$ if and only if $\rho_{1}^{i} \ll \rho_{2}^{i}$ for $i=1, \ldots, n$. 
(c) Let $\rho \in E(\mu)$. Then $\rho \in \operatorname{extr} E(\mu)$ if and only if $\rho^{i} \wedge \rho^{i^{\prime}}=0$ whenever $i \neq i^{\prime}$, where $\rho^{i}$ are as defined in (b).

(d) For every $\rho \in E(\mu)$ there exist $\pi_{j} \in \operatorname{extr} E(\mu)$ with $\rho=\sum_{j=1}^{\infty} t_{n j} \pi_{j}$.

Proof. Assertion (a) is obvious. As for (b), it is clear that the mapping in question preserves $\sigma$-convex combinations. The assertions concerning injectivity and absolute continuity follow from the formula

$$
\rho\left(\bigcup_{i=1}^{n} M_{i} \cap E_{i}\right)=\sum_{i=1}^{n} \rho^{i}\left(M_{i}\right), \text { where } M_{1}, \ldots, M_{n} \in \mathfrak{M},
$$

since

$$
\Re=\left\{\bigcup_{i=1}^{n} M_{i} \cap E_{i}: M_{1}, \ldots, M_{n} \in \mathfrak{M}\right\} .
$$

To prove the "onto" assertion, given $\rho \in \mathscr{E}_{\mu, n}$, define $\rho$ by formula (3). Observe that this definition is correct. Indeed, take $\tilde{M}_{1}, \ldots, \bar{M}_{n} \in \mathfrak{M}$ with

$$
\bigcup_{i=1}^{n} M_{i} \cap E_{i}=\bigcup_{i=1}^{n} \tilde{M}_{i} \cap E_{i}
$$

Then $\left(M_{i} \Delta \tilde{M}_{i}\right) \cap E_{i}=\varnothing$, whence $\rho^{i}\left(M_{i} \Delta \bar{M}_{i}\right)=0$, by the definition of $\mathscr{C}_{\mu, n}$. Therefore $\rho^{i}\left(M_{i}\right)=\rho^{i}\left(\tilde{M}_{i}\right)$. Since $M=\bigcup_{i=1}^{n} M \cap E_{i}$, we have $\rho(M)=\mu(M)$ for $M \in \mathfrak{M}$. The additivity of $\rho$ follows from that of $\rho^{i}, i=1, \ldots, n$. In sum, $\rho \in E(\mu)$, and we are done.

In view of (a) and (b), (c) and (d) are consequences of Theorems 1(b) and 2(b), respectively.

To prove our next theorem, we shall need two lemmas.

Lemma 1. Let $\pi, \rho \in \mathrm{ba}_{+}(\mathfrak{A})$ and let $\mathfrak{R}=\left(\mathfrak{M} \cup\left\{E_{1}, E_{2}, \ldots\right\}\right)_{b}$, where $E_{1}, E_{2}, \ldots$ are pairwise disjoint subsets of $\Omega$. Then $\pi \ll \rho$ if the following two conditions hold for $k=1,2, \ldots$ :

(i) $\pi\left|\Re_{k} \ll \rho\right| \mathfrak{R}_{k}$, where $\mathfrak{R}_{k}=\left(\mathfrak{M} \cup\left\{E_{1}, \ldots, E_{k}\right\}\right)_{b}$;

(ii) $\pi\left(M \cap\left(\bigcup_{j=1}^{k} E_{j}\right)^{c}\right) \leqslant \rho\left(M \cap\left(\bigcup_{j=1}^{k} E_{j}\right)^{c}\right)$ for $M \in \mathfrak{M}$.

Proof. Fix $\varepsilon>0$ and take $k_{0}$ with $\pi\left(\bigcup_{l=k_{0}+1}^{m} E_{l}\right)<\varepsilon / 3$ whenever $m>k_{0}$. By (i), there exists $0<\delta<\varepsilon / 3$ such that $\rho(R)<\delta$ implies $\pi(R)<\varepsilon / 3$ for all $R \in \mathfrak{R}_{k_{0}}$. Since

$$
\Re=\bigcup_{k=1}^{\infty} \Re_{k},
$$

it follows from (4) that every $R \in \Re$ can be represented in the form

$$
R=R_{1} \cup\left(\bigcup_{l=k_{0}+1}^{m} M_{l} \cap E_{l}\right) \cup\left(M \cap\left(\bigcup_{j=1}^{m} E_{j}\right)^{c}\right),
$$

where $R_{1} \in \mathfrak{R}_{k_{0}}$ and $M_{k_{0}+1}, \ldots, M_{m}, M \in \mathfrak{M}$. Taking into account our choice of $k_{0}$ and $\delta$ and applying (ii), we conclude that $\rho(R)<\delta$ implies $\pi(R)<\varepsilon$. 
Lemma 2. Let $\mu \in \mathrm{ba}_{+}(\mathfrak{M})$ and $\rho \in \mathrm{ba}_{+}(\mathfrak{M})$, and let $\mathfrak{M}=(\mathfrak{M} \cup\{E\})_{b}$, where $E \subset \Omega$. If $\Omega_{0} \in \mathfrak{W}$ is disjoint from $E$ and

(i) $\mu \ll \rho \mid \mathfrak{M}$,

(ii) $\mu\left(M \cap \Omega_{0}^{c}\right) \leqslant \rho\left(M \cap \Omega_{0}^{c}\right)$ for all $M \in \mathfrak{M}$,

then there exists $\pi \in \operatorname{extr} E(\mu)$ such that

(i) $\pi \ll \rho$,

(ii)' $\pi\left(R \cap\left(\Omega_{0} \cup E\right)^{c}\right) \leqslant \rho\left(R \cap\left(\Omega_{0} \cup E\right)^{c}\right)$ for all $R \in \mathfrak{R}$.

Proof. Since $\mathfrak{R}=\left(\mathfrak{M} \cup\left\{\Omega_{0} \cup E\right\}\right)_{b}$, it follows from (4) that (ii)' is equivalent to the condition

$$
\pi\left(M \cap\left(\Omega_{0} \cup E\right)^{c}\right) \leqslant \rho\left(M \cap\left(\Omega_{0} \cup E\right)^{c}\right) \text { for all } M \in \mathfrak{M} .
$$

It is then easy to see that we may confine ourselves to the case where $\Omega_{0}=\varnothing$. In this case (i) follows from (ii), which now reads: $\mu \leqslant \rho \mid \mathfrak{M}$. Define $E_{1}=E, E_{2}=E^{c}$ and

$$
\rho^{i}(M)=\rho\left(M \cap E_{i}\right) \text { for } \quad M \in \mathfrak{M} \text {. }
$$

We then have $\mu \leqslant \rho^{1}+\rho^{2}$. By Theorems 1(b), 2(c) and [3], Theorem 6.2.2, there exists $\pi \in \operatorname{extr} \mathscr{D}_{\mu, 2}$ with $\pi^{1} \leqslant \rho^{1}$ and $\pi^{2} \leqslant \rho^{2}$. It follows that, in the notation of Theorem 3, $\pi \in \mathscr{C}_{\mu, 2}$, and so $\pi \in \operatorname{extr} \mathscr{C}_{\mu, 2}$. It now suffices to choose $\pi \in E(\mu)$ which corresponds to $\pi$ according to Theorem $3(\mathrm{~b})$.

The following two results are motivated by [8], Theorem 2, which describes the extreme points of $E(\mu)$ with the help of various minimality conditions with respect to the relation $\ll$.

THEOREM 4. Let $\mu \in \mathrm{ba}_{+}(\mathfrak{M})$ and let $\mathfrak{R}=(\mathfrak{M} \cup \mathbb{F})_{b}$, where $\mathfrak{E} \subset 2^{\Omega}$ is a family of pairwise disjoint sets. Then for every $\rho \in E(\mu)$ there exists $\pi \in \operatorname{extr} E(\mu)$ with $\pi \ll \rho$.

Proof. We first prove the assertion under the additional assumption that $\&$ is countable, say $\mathbb{E}=\left\{E_{1}, E_{2}, \ldots\right\}$. As in Lemma 1 , define

$$
\mathfrak{R}_{k}=\left(\mathfrak{M} \cup\left\{E_{1}, \ldots, E_{k}\right\}\right)_{b}, \quad k=1,2, \ldots
$$

Using Lemma 2, we can define, by induction, $\pi_{k} \in \mathrm{ba}_{+}\left(\Re_{k}\right)$ such that

$$
\begin{gathered}
\pi_{k} \ll \rho \mid \mathfrak{R}_{k}, \quad \pi_{1} \in \operatorname{extr} E\left(\mu, \mathfrak{R}_{1}\right), \quad \pi_{k+1} \in \operatorname{extr} E\left(\pi_{k}, \mathfrak{R}_{k+1}\right), \\
\pi_{k}\left(R \cap\left(\bigcup_{j=1}^{k} E_{j}\right)^{c}\right) \leqslant \rho\left(R \cap\left(\bigcup_{j=1}^{k} E_{j}\right)^{c}\right) \text { for } R \in \mathfrak{R}_{k} .
\end{gathered}
$$

In view of (5), the $\pi_{k}$ 's admit a (unique) common extension $\pi$ to $\Re$. Clearly, $\pi \in \operatorname{extr} E(\mu)$. An application of Lemma 1 yields $\pi \ll \rho$.

To establish the general case, define

$$
\mathfrak{M}_{0}=(\mathfrak{M} \cup \mathcal{N}(\rho))_{b} \quad \text { and } \quad \mu_{0}=\rho \mid \mathfrak{M}_{0}
$$

In view of [12], Theorem 1 , we have $\mu_{0} \in \operatorname{extr} E\left(\mu, \mathfrak{M}_{0}\right)$. Put

$$
\mathfrak{F}_{0}=\{E \in \mathbb{E}: \rho(E)>0\} \text {. }
$$

Clearly, $\mathfrak{F}_{0}$ is countable and $\mathfrak{R}=\left(\mathfrak{M}_{0} \cup \mathfrak{C}_{0}\right)_{b}$. By what we have proved so far, there exists $\pi \in \operatorname{extr} E\left(\mu_{0}, \mathfrak{R}\right)$ with $\pi \ll \rho$. Of course, $\pi \in \operatorname{extr} E(\mu)$, and this proof is completed. 
We note that Theorem 4 is a partial generalization of Theorem $3(d)$. In fact, the latter theorem implies that, for finite $\&$, we can replace $\pi \ll \rho$ with $t \pi \ll \rho$ for some $t>0$. This stronger version fails if $\mathbb{E}$ is countable, as an example due to $\mathrm{R}$. Bierlein reveals ([5], pp. 95-96; cf. also Example 1 above).

THEOREM 5. Let $\mu \in \mathrm{ba}_{+}(\mathfrak{M})$ and let $\Re=\left(\mathfrak{M} \cup(\mathbb{S})_{b}\right.$, where $\mathbb{E} \subset 2^{\Omega}$ is a family of sets well ordered by inclusion. Then for every $\rho \in E(\mu)$ there exists $\pi \in \operatorname{extr} E(\mu)$ with $\pi \ll \rho$.

Proof. We assume that $\rho$ is $\sigma$-additive. This is legitimate, since, by the Stone representation theorem, we may assume that $\Omega$ is a compact space and $R$ is its algebra of open-and-closed sets. Denote by $\rho_{\beta}$ and $\mu_{\beta}$ the (unique) extensions of $\rho$ and $\mu$ to measures on $\mathfrak{R}_{\beta}$ and $\mathfrak{M}_{\beta}$, respectively ([3], Theorem 3.5.2). Then $\rho_{\beta}$ is an extension of $\mu_{\beta}$. In view of Theorem 7 below, there exists a measure

$$
\pi_{\beta} \in \operatorname{extr} E\left(\mu_{\beta}, \Re_{\beta}\right) \quad \text { with } \quad \pi_{\beta} \ll \rho_{\beta} .
$$

Since $\mu_{\beta} \in \operatorname{extr} E\left(\mu, \mathfrak{M}_{\beta}\right)$ by [3], Theorem 3.5.3, and [12], Theorem 1, it follows that $\pi_{\beta} \in \operatorname{extr} E\left(\mu, \Re_{\beta}\right)$. Putting $\pi=\pi_{\beta} \mid \Re$ and applying [12], Theorem 1, again, we conclude that $\pi$ is as desired.

Note that an analogous reduction of Theorem 4 to Theorem 7 below is possible. However, the proof we have given above avoids any uncountable form of the axiom of choice.

The following example shows that, in Theorem 5 , it is not enough to assume that $\mathbb{E}$ is linearly ordered by inclusion (even for countable (E)).

Example 2 (cf. [8], Example). Let $\Omega=[0,1)$, take $\mathfrak{M}=\{\varnothing, \Omega\}$ and define $\mu$ on $\mathfrak{M}$ by $\mu(\varnothing)=0$ and $\mu(\Omega)=1$. Let

$$
\mathfrak{E}=\{[0, t): 0<t<1 \text { is rational }\}
$$

and let $\rho$ be the restriction of Lebesgue measure to $\Re=\mathbb{E}_{b}$. Then for every $\pi \in \operatorname{extr} E(\mu)$ we have $\pi(\Re)=\{0,1\}$ (see [12], Remark 1), whence $\pi \ll \rho$ does not hold.

4. Extensions of measures. Throughout this section $M$ and $\mathfrak{M}$ stand for $\sigma$-algebras of subsets of a set $\Omega$ with $\mathfrak{M} \subset \mathfrak{R}$. The Dedekind complete vector lattice of all real-valued $\sigma$-additive functions on $\mathfrak{M}$ is denoted by ca(M) (see [3], Section 2.4; cf. also [9], Example 25.3). The elements of $\mathrm{ca}_{+}(\mathfrak{M})$ are called measures.

Given $\mu \in \mathrm{ca}_{+}(\Re)$, we denote by $E_{\sigma}(\mu, \Re)$ or $E_{\sigma}(\mu)$ the $\sigma$-convex set $E(\mu) \cap \mathrm{ca}(\Re)$. In contrast to $E(\mu)$, it may happen that $E_{\sigma}(\mu)$ is empty (see, e.g., [4], Satz 1C). This is, however, not the case in the situations we consider below, that is, when there exists a family $\& \subset 2^{\Omega}$ of pairwise disjoint sets ([3], Satz 2B, and [2], Corollary 3) or well ordered by inclusion $\left([\mathbf{1 3}]\right.$, Satz (3)) with $\mathfrak{R}=\left(\mathfrak{M} \cup(\mathfrak{S})_{\beta}\right.$.

We now give a version of Theorem 3(a) - (c) for measures. Its parts (b) and (c) are closely related with [10], pp. 311-312, [4], Satz 2A (see also [5], Theorem 1(a)), and [5], Theorem 1(b).

TheOREM 6. Let $\mu \in \mathrm{ca}_{+}(\mathfrak{M})$ and let $\mathfrak{R}=\left(\mathfrak{M} \cup\left\{E_{1}, E_{2}, \ldots\right\}\right)_{\beta}$, where $\left\{E_{1}, E_{2}, \ldots\right\}$ is a partition of $\Omega$. Define $\mathscr{E}_{\mu, \mathbb{N}}$ as

$$
\left\{\boldsymbol{p} \in \mathscr{D}_{\mu, \mathbb{N}}: M \cap E_{i}=\varnothing \text { implies } \rho^{i}(M)=0 \text { whenever } M \in \mathfrak{M}, i=1,2, \ldots\right\} \text {. }
$$


Then

(a) $\mathscr{C}_{\mu, \mathbb{N}}$ is an extreme subset of $\mathscr{D}_{\mu, \mathbb{N}}$.

(b) The mapping $\rho \rightarrow \rho$ of $\mathrm{ca}_{+}(\mathfrak{R})$ into $\left(\mathrm{ca}_{+}(\mathfrak{M})\right)^{\mathbb{N}}$, where $\rho^{i}(M)=\rho\left(M \cap E_{i}\right)$ for all $M \in \mathfrak{M}$ and $i=1,2, \ldots$, is injective, preserves $\sigma$-convex combinations and takes $E_{\sigma}(\mu)$ onto $\mathscr{C}_{\mu, \mathbb{N}}$. Moreover, $\rho_{1} \ll \rho_{2}$ if and only if $\rho_{1}^{i} \ll \rho_{2}^{i}$ for $i=1,2, \ldots$

(c) Let $\rho \in E_{\sigma}(\mu)$. Then $\rho \in \operatorname{extr} E_{\sigma}(\mu)$ if and only if $\rho^{i} \wedge \rho^{i^{\prime}}=0$ whenever $i \neq i^{\prime}$, where $\rho^{i}$ are as defined in (b).

The proof is analogous to that of Theorem 3. We only note that (3) and (4) are now replaced by

$$
\begin{gathered}
\rho\left(\bigcup_{i=1}^{\infty} M_{i} \cap E_{i}\right)=\sum_{i=1}^{\infty} \rho^{i}\left(M_{i}\right), \text { where } M_{1}, M_{2}, \ldots \in \mathfrak{M}, \\
\Re=\left\{\bigcup_{i=1}^{\infty} M_{i} \cap E_{i}: M_{1}, M_{2}, \ldots \in \mathfrak{M}\right\},
\end{gathered}
$$

respectively.

THEOREM 7. Let $\mu \in \mathrm{ca}_{+}(\mathfrak{M})$ and let $\mathfrak{R}=(\mathfrak{M} \cup \mathfrak{F})_{\beta}$, where $\mathfrak{F} \subset 2^{\Omega}$ is a family which either consists of pairwise disjoint sets or is well ordered by inclusion. Then for every $\rho \in E_{\sigma}(\mu)$ there exists $\pi \in \operatorname{extr} E_{\sigma}(\mu)$ with $\pi \ll \rho$.

Proof. We first deal with the case where $\&$ consists of pairwise disjoint sets. It is then possible to derive the assertion from Theorem 4 with the help of [12], Theorem 1, and a classical extension theorem ([3], Theorems 3.5.2 and 3.5.3). Alternatively, for countable (5) the assertion follows from Theorems 1(b) and 2(a) with the help of Theorem $6(\mathrm{~b})$ and [3], Theorem 6.2.2. One has only to observe that if $\rho \in \mathscr{E}_{\mu, \mathbb{N}}$ and $\pi \in \mathscr{D}_{\mu, \mathbb{N}}$ satisfy $\pi^{i} \ll \rho^{i}$ for all $i$, then $\pi \in \mathscr{E}_{\mu, \mathbb{N}}$. To establish the general case, it is enough to apply the reduction argument of the proof of Theorem 4.

Now we deal with the case where $\mathbb{E}$ is well ordered by inclusion $\subset$. For countable $\mathbb{E}$ this case reduces to the previous one. Indeed, define $\mathfrak{F}=\left\{F_{E}: E \in \mathbb{E}\right\}$, where

$$
F_{E}=E \backslash \bigcup\left\{E^{\prime} \in \mathbb{E}: E^{\prime} \subsetneq E\right\} \text {. }
$$

Then $\mathfrak{F}$ is a countable family of pairwise disjoint sets and $\mathfrak{F}_{\beta}=\mathfrak{F}_{\beta}$. Therefore $\mathfrak{M}=(\mathfrak{M} \cup \mathfrak{\Re})_{\beta}$

For arbitrary $\mathbb{E}$ it is enough to find a countable subfamily $\mathfrak{E}_{0}$ such that

$$
\mathfrak{R}=\left(\mathfrak{M} \cup \mathcal{N}(\rho) \cup \mathfrak{F}_{0}\right)_{\beta},
$$

and then argue as in the final part of the proof of Theorem 4. Observe that $\rho(\mathbb{E})$ is a well-ordered subset of $\mathbb{R}$, and so it is countable. Therefore $\rho(\mathbb{E})=\rho\left(\mathbb{E}_{0}\right)$ for some countable $\mathfrak{E}_{0} \subset \mathbb{E}$. It follows that for every $E \in \mathbb{E}$ there exists $E_{0} \in \mathbb{E}_{0}$ such that $\rho(E)=\rho\left(E_{0}\right)$ and $E \subset E_{0}$ or $E_{0} \subset E$. Hence $E \in\left(\mathcal{N}(\rho) \cup \mathscr{E}_{0}\right)_{b}$, and we are done.

A straightforward modification of Example 2 shows that, in Theorem 7, it is not enough to assume that $\mathbb{E}$ is linearly ordered by inclusion (even for countable $\mathbb{S}$ ). We shall present another example to the same effect with the additional property that $\operatorname{extr} E_{\sigma}(\mu) \neq$ $\varnothing$ for every $\mu \in \mathrm{ca}_{+}(\mathfrak{M})$. (That this property holds below follows from [2], Corollary 4 and its proof, and [12], Theorem 1.) 
EXAMPLE 3 . Let $\Omega=[0,1] \times[0,1]$ and

$$
\begin{aligned}
\mathfrak{M} & =\{B \times[0,1]: B \text { is a Borel subset of }[0,1]\}, \\
\mathfrak{E} & =\{[0,1] \times[0, t]: 0<t<1 \text { is rational }\} .
\end{aligned}
$$

Then $\mathfrak{R}=(\mathfrak{M} \cup \mathfrak{C})_{\beta}$ is the Borel $\sigma$-algebra of $\Omega$. Let $\mu$ and $\rho$ be the restrictions of the two-dimensional Lebesgue measure to $\mathfrak{M}$ and $\mathfrak{R}$, respectively. If $\pi \in \operatorname{extr} E_{\sigma}(\mu)$, then, as is well known, $\pi$ is concentrated on the graph $G_{f}$ of a Borel function $f:[0,1] \rightarrow[0,1]$. On the other hand, $\rho\left(G_{f}\right)=0$, and so $\pi \ll \rho$ does not hold.

Postscript. For results related to Theorem 2(b) see Z. Lipecki, On binary-type approximations in vector lattices (submitted).

\section{REFERENCES}

1. C. D. Aliprantis and O. Burkinshaw, Positive operators (Academic Press, 1985).

2. A. Ascherl and J. Lehn, Two principles for extending probability measures, Manuscripta Math. 21 (1977), 43-50.

3. K. P. S. Bhaskara Rao and M. Bhaskara Rao, Theory of charges. A study of finitely additive measures (Academic Press, 1983).

4. D. Bierlein, Über die Fortsetzung von Wahrscheinlichkeitsfeldern, Z. Wahrsch. Verw. Gebiete 1 (1962), 28-46.

5. D. Bierlein and W. J. A. Stich, On the extremality of measure extensions, Manuscripta Math. 63 (1989), 89-97.

6. D. A. Edwards, On a theorem of Dvoretsky, Wald, and Wolfowitz concerning Liapounov measures, Glasgow Math. J. 29 (1987), 205-220.

7. H. Goller, An extension of Lyapounov's convexity theorem and (non-) randomization of tests, Statist. Decisions 2 (1984), 315-328. 288-293.

8. Z. Lipecki, On extreme extensions of quasi-measures, Arch. Math. (Basel) 58 (1992),

9. W. A. J. Luxemburg and A. C. Zaanen, Riesz spaces, Vol. I (North-Holland, 1971).

10. O. Nikodym, Sur les fonctions d'ensembles, in Comptes-Rendus du I Congrès des Mathématiciens des Pays Slaves, Warszawa 1929 (Warszawa 1930), 304-313.

11. D. Oates, A sequentially convex hull, Bull. London Math. Soc. 22 (1990), 467-468.

12. D. Plachky, Extremal and monogenic additive set functions, Proc. Amer. Math. Soc. 54 (1976), 193-196. $157-159$.

13. H. Weber, Ein Fortsetzungssatz für gruppenwertige Masse, Arch. Math. (Basel) 34 (1980),

INSTITUTE OF MATHEMATICS

Polish ACademy of Sciences

Wroclaw BRANCH

KOPERNIKA 18

51-617 WroCŁAW

POLAND 